\title{
Measurement Error in Estimated GFR Slopes across Transplant Chronic Kidney Disease Stages
}

\author{
Mohammad Akhtar Hossain Ahmad Attia Ahmed Shoker \\ Saskatchewan Transplant Program, St. Paul's Hospital, Saskatoon, Sask., Canada
}

\section{Key Words}

Cockcroft-Gault $\cdot$ Modified diet in renal disease .

Glomerular filtration rate

\begin{abstract}
Background: This study examines if transplant glomerular filtration rate (GFR) slope prediction is affected by the degree of transplant chronic kidney disease (CKDT) stage. Methods: Serial changes in estimated GFR ( $\triangle$ eGFR) by Cockcroft-Gault (CG) and Modified Diet in Renal Disease-Isotope Dilution Mass Spectrometry (MDRD-IDMS) equations were compared to simultaneous changes in isotope GFR ( $\Delta \mathrm{i} G F R)$ in renal transplant patients who had at least four scans. $R \boldsymbol{e}$ sults: Total number of patients (iGFR scans) was 99 (772) while the corresponding numbers in CKDT stages 1-4 were 33 (103), 69 (239), 75 (316) and 37 (96), respectively. Measurement error $[(\Delta \mathrm{eGFR}-\Delta \mathrm{iGFR}) \times 100 / \Delta \mathrm{iGFR}]($ median $\pm \mathrm{IQR}$, interquartile range) estimated from CG and MDRD-IDMS slopes were $-414.29 \pm 276.16 \%$ and $-342.86 \pm 210.18 \%$ (stage 1); $-350.00 \pm 301.22 \%$ and $-300.00 \pm 525.00 \%$ (stage 2); $-26.02 \pm 404.38 \%$ and $-26.58 \pm 423.13 \%$ (stage 3 ); 10.26 $\pm 142.18 \%$ and $-76.92 \pm 145.64 \%$ (stage 4 ), respectively. The proportion of patients with CG measurement error $\leq 1$-fold in stages 1 and 2 of 12 and $14.5 \%$ was significantly $(p<0.05)$ lower than that of 36.3 and $52.8 \%$ at stages 3 and 4 , respectively. Similar measurement errors were observed for MDRD-
\end{abstract}

IDMS. Conclusions: Transplant GFR slope prediction is affected by the degree of renal dysfunction. Errors in slope prediction are much higher in those with better function and thus add another limitation for eGFR use in longitudinal studies on progressive graft dysfunction.

Copyright $\odot 2009$ S. Karger AG, Basel

\section{Introduction}

There can be little doubt that sequential measurements of glomerular filtration rate (GFR) must form the basis for the study of chronic allograft failure [1-3]. In clinical trials to date, GFR is estimated at multiple time points, and curves constructed from those points reflect the change in renal function over time [4-8]. In most cases, constant slopes over time are assumed, and mean slopes are compared between the study groups to evaluate the effect of interventions on the rate of GFR decline.

There are reasons to raise concerns on the validity of that approach for measuring GFR slopes. Many investigators have stressed the general limitations of estimated GFR (eGFR) equations [2] and the performance of such equations has been demonstrated to vary in patients with different degrees of renal dysfunction [9]. Indeed, several researchers have documented the substantial variability

\section{KARGER}

Fax +4161306 1234 E-Mail karger@karger.ch www.karger.com
(C) 2009 S. Karger AG, Basel

0250-8095/10/0312-0151\$26.00/0

Accessible online at:

www.karger.com/ajn
Dr. Ahmed Shoker, MD, FRCP (C)

Department of Medicine, Division of Nephrology, University of Saskatchewan 103 Hospital Drive

Saskatoon, SK S7N 0W8 (Canada)

Tel. +1 306966 2630, Fax +1 306966 7996, E-Mail ass787@ mail.usask.ca 
of the current GFR equations at different time points after transplantation $[10,11]$. It was suggested that these equations were less accurate within the first year of transplantation and that they were quite limited in assessing GFR decline over time $[12,13]$. Previously, we examined the accuracy to estimate rates of GFR decline in renal transplant patients [14]. A total of 508 isotope GFR (iGFR) tests performed on 72 patients were analyzed. We noted that eGFR predicts the iGFR with a high sensitivity but low specificity. Accuracy to predict $\Delta$ iGFR from the eGFRs was limited to less than $65 \%$ concordance within $30 \%$ range from changes in iGFR. In our previous work there was no attempt to extend our observation to determine whether there is difference in the magnitude of error to estimate rates in decline in GFR between transplant chronic kidney disease (CKDT) stages.

Taken together, this challenged us to explore the question whether limitations of eGFR estimators translate into inconsistencies in quantitating progression in CKDT in patients at different CKDT stages.

To achieve our goal, we examined the use of the Cockcroft-Gault (CG) and Modified Diet in Renal DiseaseIsotope Dilution Mass Spectrometry (MDRD-IDMS) to estimate rates of decline in renal transplant GFR across CKDT stages. To do so, we first constructed GFR slopes from serial changes in measured and estimated GFRs and then compared measurement error using changes in iGFR slopes as the reference test.

\section{Materials and Methods}

\section{Study Population}

We collected 772 matched pairs of serial iGFR and serum creatinine measurements over a follow-up duration of $7.61 \pm 6.68$ years to perform regression analysis of measured and estimated GFR over time. Least-squares fit was used to compare the performances of GFR equations. The protocol for this retrospective study was approved by the ethics board of this institution. Blood was drawn on the day of the radioisotope clearance for routine blood work including the testing of serum creatinine. In addition, patients' demographics were recorded including heights and weights. We used the same inclusion criteria published recently with minor modifications. We screened 1,400 iGFR measurements performed in our center on 345 patients followed at our clinic. With these inclusion criteria in mind, 772 iGFR studies performed on 99 patients were eligible for analysis. All recipients were adult with stable renal function that underwent primary or repeated transplantation and had at least four independent iGFR studies starting within the first year of transplantation, but at least 2 weeks from transplantation date. We defined stable renal function by lack of more than $10 \%$ change in serum creatinine within the last 4 weeks prior to the study.
During this period, there was no change in either the laboratory creatinine reference levels or the nuclear medicine protocols used. All studies were performed during the regular outpatient visits. The iGFR was considered the reference GFR. eGFR estimation was calculated from two equations: CG [15], and MDRDIDMS [16].

\section{Methods}

GFR Estimation by Isotope Scan

GFR was calculated from the disappearance of radioactivity in three timed plasma samples after a single injection of ${ }^{99 \mathrm{~m}} \mathrm{Tc}$ DTPA. This method is precise, reproducible and is independent of variations in the volume distribution of the isotope [17]. Details of the methods of eGFRs measurements were presented previously [14]. Based on the numerical value of each GFR test, the data were stratified into the current K/DOQI (CKDT) stages. Because of the lower number of scans of less than $15 \mathrm{ml} / \mathrm{min} / 1.73 \mathrm{~m}^{2}$, analysis of stage 5 was excluded. All isotope and CG eGFRs were presented corrected to $1.73 \mathrm{~m}^{2}$ after normalization for body surface area (BSA) using the Dubois-Dubois formula [18]. Thus, all pertinent comparisons are presented after normalization. Of note, MDRD-IDMS equation is already corrected for surface area and requires no measurement of additional normalization.

Measurement of Serum Creatinine Concentrations

Serum creatinine was measured with an enzymatic assay (SYNCHRON LX 20 Systems, Bachman, Coulter, Inc., Fullerton, Calif., USA) with a normal range of $60-110 \mathrm{mmol} / \mathrm{l}$.

The intra- and inter-assay CV were $<3 \%$. This method has a small bias of $\leq 6 \mu \mathrm{mol} / \mathrm{l}$ as compared to the isotope dilution mass spectrometry. However, this difference is not considered significant in the estimation of GFR [19].

Repeated Measure Analysis

A repeated measured design was accounted for measurement on GFR tests at varying times after transplantation for each subject. An exponential model was used to account for the nonlinearity of the data over time (as published previously). Assessment of within-patient variability was performed using our previously published methods [14]. The variability of individual measurements of iGFR and eGFR was evaluated by estimating the withinpatient variance components for the individual iGFR or eGFR measurements under the exponential model. The within-patient variance describes the variation within the same patients over time after accounting for long-term systematic changes as reflected in the individual patients' slopes. The estimated half-life of the graft function was calculated from the formula: (Ln2)/(-mean slope). To remove in addition the effects of a gradual change in the rate of decline over time, we also estimated the within-patient variance components under the exponential model. In addition, to obtain a better comparison of estimated slopes across stages, nonparametric comparisons were performed between the root mean square error (RMSE) of the eGFR curves of each equation separately among the studied CKDT stages.

Measurement Error in Estimating GFR Slopes

Measurement error was calculated from the equation: [(\% annual change in eGFR $-\%$ annual change in iGFR) $\times 100$ divided by $\%$ annual change in iGFR]. Comparison ( $p$ value) between measurement errors was performed using Wilcoxon signed-rank test. 
We also analyzed the distribution of error towards the measured value with non-parametric kernel density estimators [20, 21] (which is a nonparametric way of estimating the probability density function of a random variable) and a multivariable linear regression (the difference between kernel curves was judged with a repeated measure analysis of variants).

Finally, comparisons between the $\%$ of populations with measurement error of equal or less than 1-fold to those with higher ranges of error among the CKDT stages were done by the Wilcoxon signed-rank test. Univariate and multivariable analyses were performed to determine factors that may affect magnitude of errors.

All statistical evaluations were performed for the total group as well as performed separately for scans within different stages of CKDT. Continuous data is presented as mean \pm SD (except if otherwise mentioned) and discrete variables as frequency (\%). In all cases $\mathrm{p}<0.05$ was considered statistically significant. We used the Statistical Package of Social Science, SPSS 16 (SPSS Inc., Chicago, Ill., USA), MedCalc 9.5.0.0 (MedCalc Software, Mariakerke, Belgium), Systat 12.0 (Systat Software Inc., Chicago, Ill., USA) and MS Excel to perform the analysis.

\section{Results}

\section{Descriptive Statistics}

There was a wide range of renal function in the CKDT patients with Scr ranges from 0.57 to $8.69 \mathrm{mg} / \mathrm{dl}$ with a mean $\pm \mathrm{SD}$ of $1.70 \pm 0.85 \mathrm{mg} / \mathrm{dl}$. The data set consisted of 63 male and 36 female patients with a total of 772 iGFR scans. Patients were on prednisone, a calcineurin inhibitor, and mycophenolate derivative, or azathioprine. Cohort patients' demographics are summarized in table 1. Our population was comprised mainly of middle- and old-aged individuals. As expected, the data set was not normally distributed ( $\mathrm{p}$ value from Kolmogorov-Smirnov tests was $<0.05$ ), with the majority of GFR $<90 \mathrm{ml} /$ $\min / 1.73 \mathrm{~m}^{2}$. The mean reference iGFR of a total of 772 scans was $58.49 \pm 27.23 \mathrm{ml} / \mathrm{min} / 1.73 \mathrm{~m}^{2}$.

A total of $12.5 \%$ ( $\mathrm{n}$ scans $=96$ ) had iGFR between $\geq 15$ and $<30 \mathrm{ml} / \mathrm{min} / 1.73 \mathrm{~m}^{2}$ (stage 4$), 41 \%(\mathrm{n}=316)$ had $\mathrm{iGFR}$ between $\geq 30$ and $<60 \mathrm{ml} / \mathrm{min} / 1.73 \mathrm{~m}^{2}$ (stage 3 ), $31 \%$ ( $\mathrm{n}=239$ ) had iGFR between $\geq 60$ and $<90 \mathrm{ml} / \mathrm{min} / 1.73$ $\mathrm{m}^{2}$ (stage 2), and 13.5\% $(\mathrm{n}=103)$ had $\mathrm{iGFR} \geq 90 \mathrm{ml} /$ $\min / 1.73 \mathrm{~m}^{2}$ (stage 1 ). We had a small number of iGFR $(2 \%, \mathrm{n}=18)$ in stage $5\left(<15 \mathrm{ml} / \mathrm{min} / 1.73 \mathrm{~m}^{2}\right)$ and therefore these were excluded from slope analyses.

The first iGFR was performed at a mean duration of $2.66 \pm 4.07$ years from the time of transplantation. Sixteen patients had 4 scans while 26 patients had 5-6 scans, 35 patients had 7-10 scans and 22 patients had 11-16 scans. The mean number of iGFR/patient was $8 \pm 3$ scans. The mean duration between each 2 scans is shown in ta-
Table 1. Patients' demographics* $(n=99,772$ observations)

\begin{tabular}{|c|c|}
\hline & Mean $\pm \mathrm{SD}$ \\
\hline Age & $48.22 \pm 14.44$ \\
\hline Height, cm & $169.35 \pm 8.98$ \\
\hline Weight, kg & $77.63 \pm 16.23$ \\
\hline BSA & $1.88 \pm 0.22$ \\
\hline Scan/patient & $8 \pm 3$ \\
\hline Duration, years & $7.61 \pm 6.68$ \\
\hline Duration between two consecutive scans, years & $2.13 \pm 2.56$ \\
\hline Serum creatinine, $\mathrm{mg} / \mathrm{dl}$ & $1.70 \pm 0.85$ \\
\hline Isotope $\mathrm{GFR}, \mathrm{ml} / \mathrm{min} / 1.73 \mathrm{~m}^{2}$ & $58.49 \pm 27.23$ \\
\hline Estimated $\mathrm{GFR}_{\mathrm{CG}}, \mathrm{ml} / \mathrm{min} / 1.73 \mathrm{~m}^{2}$ & $58.54 \pm 23.19$ \\
\hline Estimated GFR ${ }_{\text {MDRD-IDMS }}, \mathrm{ml} / \mathrm{min} / 1.73 \mathrm{~m}^{2}$ & $51.26 \pm 22.18$ \\
\hline
\end{tabular}

\section{CKDT stages $^{\mathrm{a}}$}

Isotope $\mathrm{GFR}_{\mathrm{CKDT}}$ stage 1

Isotope $\mathrm{GFR}_{\mathrm{CKDT}}$ stage 2

* Among the 99 patients, number of males and females were 63 and 36 , respectively.

${ }^{\text {a }}$ Corresponding numbers of patients in stages $1-4$ were 33, 69, 75 and 37 , respectively.

ble 1 . The change in iGFR during the study duration was $-12.96 \mathrm{ml} / \mathrm{min} / 1.73 \mathrm{~m}^{2}$. There was a significant correlation $(\mathrm{p}<0.05)$ between the GFR performed at first observation and that obtained at the conclusion of the study.

\section{Results of Repeated Measures Analysis to Estimate \\ Rate of Decline in GFR in the Total Transplant Population}

There was a gradual and statistically significant difference $(p<0.05)$ between rates of decline in the eGFRs and iGFR in the analysis of the un-normalized GFR data set (data available on request). After normalization, the statistical difference for both equations disappeared. Parallel slope analyses on the total data set corresponded to an annual percent change in iGFR, CG and MDRD-IDMS of $-6.02 \%$ (95\% CI -3.56 to -9.58$),-6.23 \%$ (-3.76 to $-9.99)$, and $-6.82 \%$ ( -3.97 to -10.79$)$, respectively. The estimated half-lives of graft function from slope analysis showed a half-life of 11.51 years for iGFR, 11.12 years from CG, and 10.17 years from MDRD-IDMS. The extrapolated intercept from the iGFR was $81.68 \pm 76.42$ $\mathrm{ml} / \mathrm{min} / 1.73 \mathrm{~m}^{2}$. The estimated intercepts from the CG 

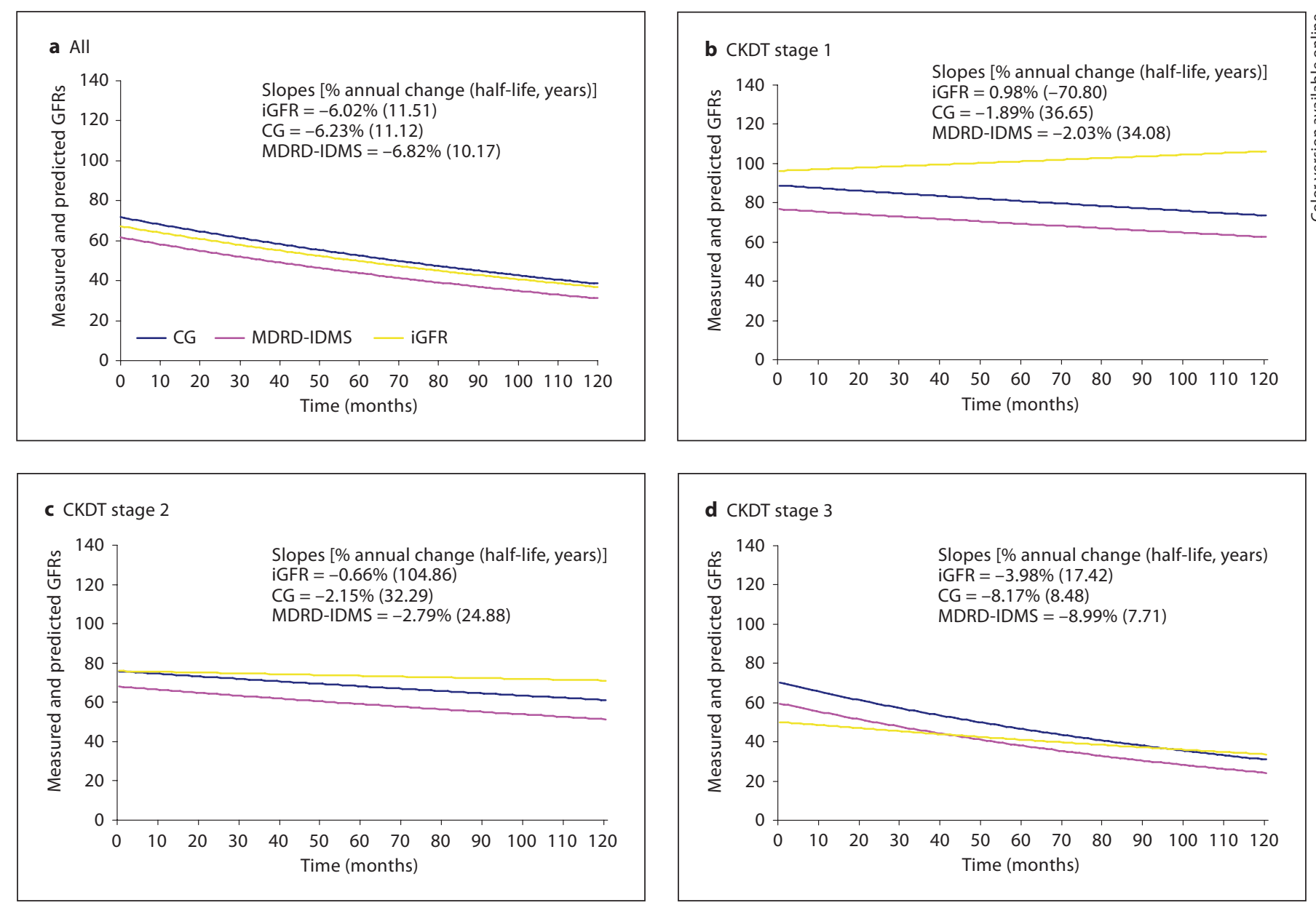

Fig. 1. Repeated measure analysis of iGFR and eGFRs showing the change of transplant renal function over a follow-up duration of $7.61 \pm 6.68$ years. Mean duration between each two consecutive observations was $2.13 \pm 2.56$ years. Whereas the slope of iGFR was not significantly different $(p>0.05)$ from CG and MDRDIDMS slopes in the overall population, the slope of iGFR was significantly different $(\mathrm{p}<0.05)$ from CG and MDRD-IDMS slopes across all stages (except in stage 4 for MDRD-IDMS). Isotope and CG GFRs were normalized to $\mathrm{ml} / \mathrm{min} / 1.73 \mathrm{~m}^{2}$. Slopes were constructed from iGFR data set of each CKDT stages separately.

and MDRD-IDMS were $88.23 \pm 107.04 \mathrm{ml} / \mathrm{min} / 1.73 \mathrm{~m}^{2}$ and $80.57 \pm 117.90 \mathrm{ml} / \mathrm{min} / 1.73 \mathrm{~m}^{2}$, respectively. All estimated intercepts were statistically different from those extrapolated from the iGFR slope when compared by the Wilcoxon signed-rank test.

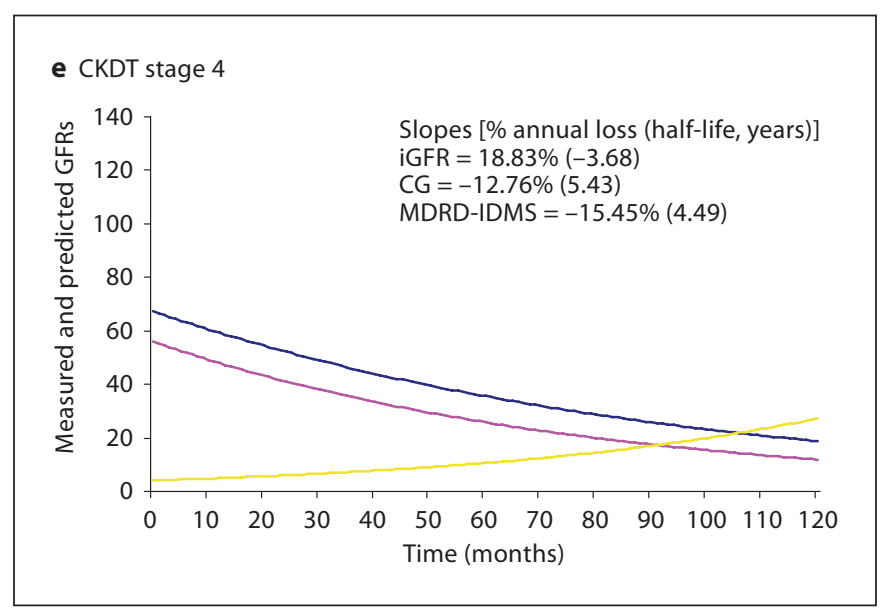

\section{Comparison of Percent Annual Changes and}

Half-Lives in iGFR and eGFRs in CKDT Stages 1-4

Further analysis was performed only on normalized data set as demonstrated by figure 1 . In stage 1 , we had a mean number of $3.12 \pm 2.9$ scans/patient. In stage 2 , the 
Table 2. Variability (RMSE) of iGFR and eGFR slopes at different CKDT stages and their comparisons

\begin{tabular}{|c|c|c|c|c|c|}
\hline \multicolumn{4}{|l|}{ RMSE } & \multicolumn{2}{|c|}{$\begin{array}{l}\mathrm{p} \text { values between } \\
\text { RMSEs of iGFR and } \\
\text { eGFRs slopes at each } \\
\text { CKDT stage* }\end{array}$} \\
\hline $\begin{array}{l}\text { CKDT } \\
\text { stages }\end{array}$ & iGFR & CG & MDRD-IDMS & $\begin{array}{l}\text { iGFR } \\
\text { vs. CG }\end{array}$ & $\begin{array}{l}\text { iGFR vs. } \\
\text { MDRD-IDMS }\end{array}$ \\
\hline Stage 1 & $2.70 \pm 3.85$ & $2.79 \pm 4.03$ & $2.69 \pm 3.82$ & 0.51 & 0.92 \\
\hline Stage 2 & $2.51 \pm 4.22$ & $2.49 \pm 2.94$ & $2.29 \pm 3.59$ & 0.51 & 0.50 \\
\hline Stage 3 & $1.91 \pm 2.40$ & $2.40 \pm 2.74$ & $2.09 \pm 2.63$ & 0.07 & $<0.05$ \\
\hline Stage 4 & $0.93 \pm 1.95$ & $0.90 \pm 1.38$ & $1.14 \pm 2.19$ & 0.65 & $<0.05$ \\
\hline
\end{tabular}

* p value from the Wilcoxon signed-rank test measured at the 0.05 level of significance.

mean number of scans was $3.46 \pm 2.6 /$ patient. The mean number of scans in stage 3 was $3.95 \pm 2.8$ /patient. In stage 4 , we had a mean number of scans of $2.67 \pm 1.7 / \mathrm{pa}-$ tient. Figure 1 presents also the $\%$ annual change in iGFR and eGFRs by CKDT stages. Of note, a negative denotes loss of function while positive reflects gain of function. As such, both the MDRD-IDMS and CG consistently overestimated graft loss in the four stages as seen in figure 1. In addition, there was directional error in the estimating equations in stages 1 and 4 as well. Whereas iGFR slope was not significantly different $(p>0.05)$ from that of CG and MDRD-IDMS in the overall population, iGFR slope was significantly less steep $(p<0.05)$ from the estimated slopes across all stages (except in stage 4 for MDRD-IDMS). There was also a clear difference between annual losses in GFRs and the corresponding half-lives in CKDT stage analyses. For example, the annual change in iGFR in stage 1 was $0.98 \%$ (95\% CI of 2.04 to -0.09 ) with a corresponding half-life of -70.80 years. In stage 3 , however, the annual change in iGFR slope was $-3.98 \%$ (95\% CI of -1.26 to -5.24$)$, with a half-life of 17.42 years. $\mathrm{p}$ values between \% annual change in estimated and measured GFRs were statistically significant throughout the stages (except between iGFR and MDRD-IDMS in stage $4)$.

\section{Variability (RMSE) in Slope Predictions}

Table 2 lists the respective RMSE of slopes of iGFR and eGFRs at different CKDT stages and their comparisons. After normalization of iGFR, there was no significant difference in variability between estimated and measured slopes except for higher variability of MDRD-IDMS dur- ing stages 3 and 4 . It should be noted that before normalization, variability in iGFR slopes were significantly higher as reported previously [14]. In general RMSE for both estimators was relatively higher, while that for iGFR was lower in CKDT stages 3 and 4.

\section{Measurement Error in Estimating GFR Slopes across CKDT Stages 1-4}

Table 3 presents the results of measurement errors. There was a tendency for a higher magnitude of error in early CKDT stages. For example, CG measurement error in stage 1 was higher than 4 -fold. The magnitude of error in GFR in stage 4 was $10 \%$ from the CG equation. MDRDIDMS measurement error overestimated the annual GFR changes in all stages with an obvious wider range of error in those with relatively better function. The magnitude of error in estimating GFR slopes was variable and frequently significant between some, but not all stages. For example, the range of error in CG estimated slope for CKDT stage 1 was significantly higher than that in stage 4 , while it was not significantly different from that in stages 2 or 3. Similarly, the error in estimating MDRDIDMS slopes in stage 2 was significantly higher than that in stage 3 or 4 . Figure $2 a, b$ depicts graphically the distribution of measurement error in the estimation of GFR slopes. The solid curves shown represent the magnitude of error in GFR slope estimation. A trend towards overestimation of progression in transplant dysfunction was observed. Analyses using nonparametric kernel density estimator for measurement error in eGFR slopes demonstrated significant difference in the magnitude of error between most of its stages. Notably, there was no difference in slopes between stage 1 versus stage 2 or 3 and between stage 3 and 4 in MDRD-IDMS estimated slopes. Figure $3 \mathrm{a}, \mathrm{b}$ shows the proportions of patients with narrow and wide margins of measurement errors. The proportions of patients with estimated errors of $\leq 1$-fold for CG were $12.5,14.5,36.3$ and $52.8 \%$ for stages $1-4$, respectively. The estimation errors of more than 1 -fold to less or equal to 2 -fold for the same equation for the same stages, respectively, were $18.2,10.1,16.3$ and $19.4 \%$. The proportions of patients with estimated errors of more than 2fold from the same equation were 69.7, 75.4, 47.5 and $27.85 \%$ for stages $1-4$, respectively. The proportion of patients with CG measurement error $\leq 1$-fold in stages 1 and 2 was significantly $(\mathrm{p}<0.05)$ lower than that at stages 3 and 4, respectively. Similar proportions of error were observed for MDRD-IDMS.

Finally, univariate and multivarible analyses were employed to determine demographic factors that may affect 


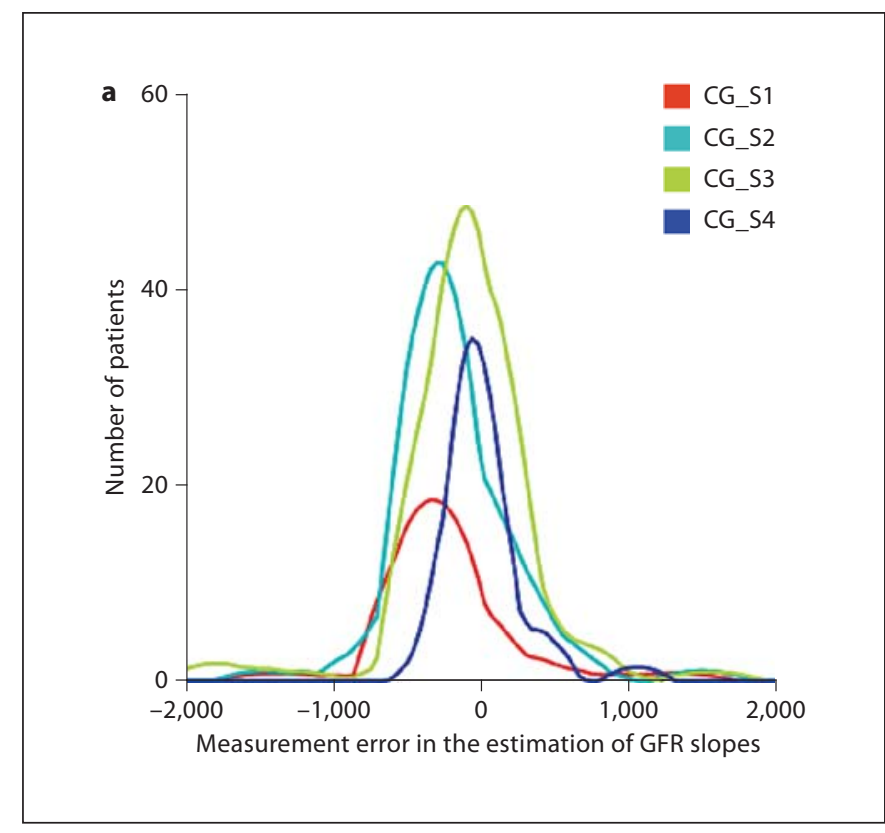

Fig. 2. Nonparametric kernel density estimator (analogous to a continuous histogram that shows where the data are most concentrated in the sample) showing the distribution of errors in the estimation of CKDT slopes from eGFRs. Measurement error was

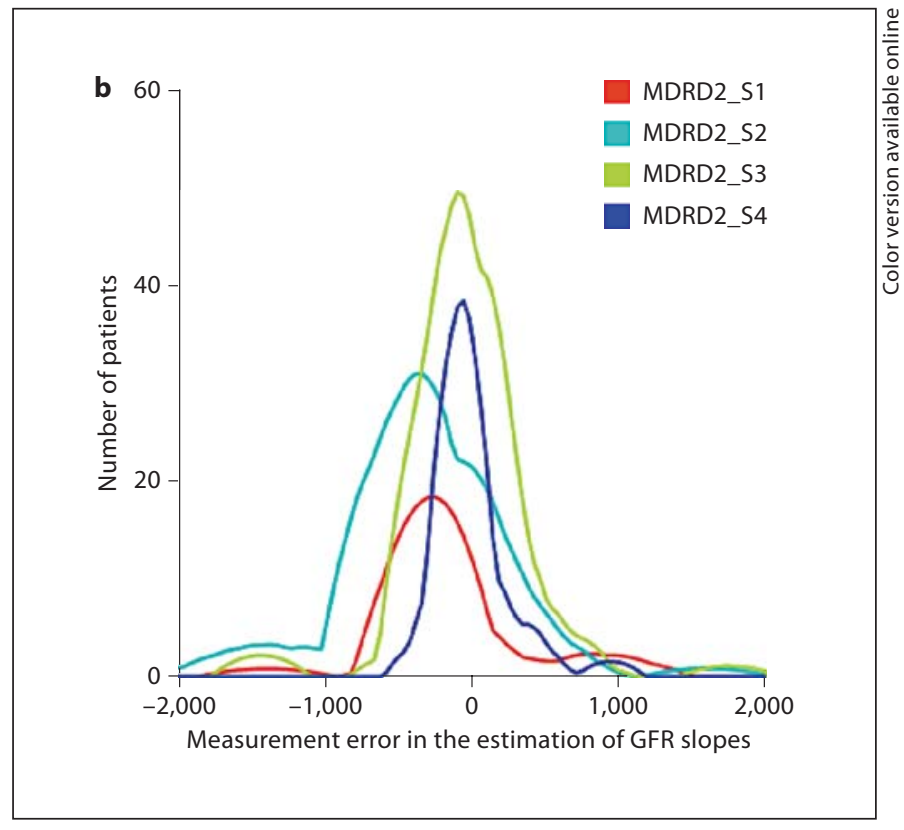

defined as (\% annual change in eGFR - $\%$ annual change in iGFR) divided by $\%$ annual change in iGFR. The errors were significantly different $(\mathrm{p}<0.05)$ among stages for both eGFRs (except S1 vs. $\mathrm{S} 2 / \mathrm{S} 3, \mathrm{~S} 3$ vs. S4 for both estimators). S = CKDT stage.

Table 3. Measurement error $^{\mathrm{a}}\left(\right.$ Median $\left.\pm \mathrm{IQR}^{\mathrm{b}}\right)$ in estimating GFR slopes

\begin{tabular}{|c|c|c|c|c|c|c|c|c|c|c|}
\hline & \multicolumn{4}{|c|}{ Measurement error at different CKDT stages (\%) } & \multicolumn{6}{|c|}{$\mathrm{p}$ values between measurement errors between CKDT stages* } \\
\hline & 1 & 2 & 3 & 4 & S1 vs. S2 & S1 vs. S3 & S1 vs. S4 & S2 vs. S3 & S2 vs. S4 & S3 vs. S4 \\
\hline CG & $-414.29 \pm 276.16$ & $-350.00 \pm 301.22$ & $-26.02 \pm 404.38$ & $10.26 \pm 142.18$ & 0.94 & 0.05 & $<0.05$ & 0.04 & $<0.05$ & 0.26 \\
\hline MDRD-IDMS & $-342.86 \pm 210.18$ & $-300.00 \pm 525.00$ & $-26.58 \pm 423.13$ & $-76.92 \pm 145.64$ & 0.09 & 0.15 & 0.15 & $<0.05$ & $<0.05$ & 0.59 \\
\hline
\end{tabular}

* $\mathrm{p}$ value from Wilcoxon Signed Rank test measured at the 0.05 level of significance.

${ }^{\mathrm{a}}$ Measurement error $=[(\Delta \mathrm{eGFR}-\Delta \mathrm{iGFR}) \times 100 / \Delta \mathrm{iGFR}] .{ }^{\mathrm{b}} \mathrm{IQR}=$ Interquartile range.

range of error in estimating GFR slopes. Univariate analysis of the predictive role of different variables on measurement error to estimate GFR slopes was performed using a binary logistic regression model. Measurement errors below or equal to 1 -fold and more than 1-fold across stages were considered binary outcomes in the logistic regression model. The following factors were assessed: age, gender, height, weight, BMI, serum creatinine, iGFR and eGFRs values, intercepts and \% annual change in GFR. None of the tested factors showed statistical significance.

\section{Discussion}

This current project is the first, to our knowledge, to focus on how GFR slope predictions are affected by the degree of renal dysfunction. It should be emphasized that the objective of this analysis was not to present rigorous analysis of longitudinal graft function outcomes but to study the effect of renal dysfunction on the goodness of eGFR slopes as a mathematical tool for the study of rate of decline in renal function. Therefore, patient attrition or variation in individual outcomes was not expected to affect the mathematical analysis. 

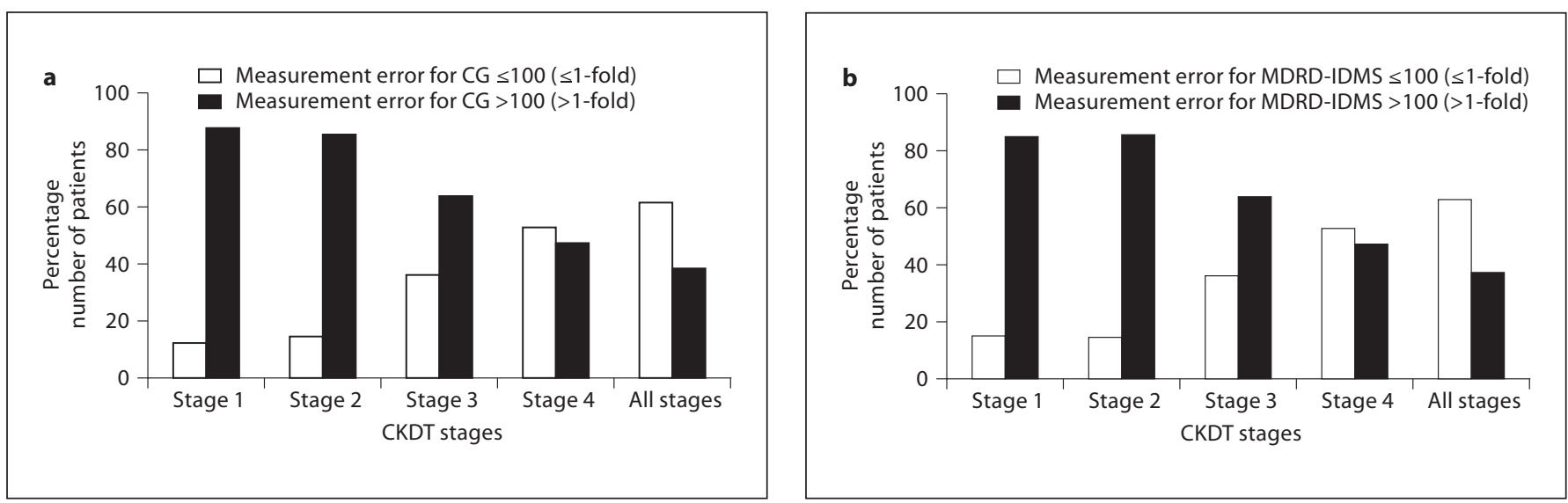

Fig. 3. Distribution of measurement error below or equal to 1 -fold versus more than 1 -fold across stages. For both eGFR estimators, p values were $<0.05$ between: (1) stage 1 vs. $3 / 4$; (2) stage 2 vs. 3/4; (3) all stages vs. stages 1 and 2 .

Among the equations tested, we selected the most commonly used two equations, CG and MDRD-IDMS. There was no attempt, however, to study the influence of renal function on slope analysis. In this paper, we extended the study to a total of 99 patients with a total of 772 measurements across CKDT stages.

With this in mind, the results raise several points for discussion. First, we as well as Gera et al. [10] have demonstrated the relatively low precision and accuracy of eGFR slopes in transplant patients. In our previous work on 72 patients, we reported a high sensitivity, less withinpatient variability but low specificity of the CG and MDRD-IDMS equations to estimate rates of decline in transplant GFR. In addition, we noted a significantly lower percent annual loss in mean iGFR of $6.03 \%$ than that of CG of $8.62 \%$ or MDRD2 of $8.96 \%$ [14]. Of note, 331 isotope scans on 46 patients from a previous study were included in this current study. An additional major difference between these two studies is the analysis of each CKDT stage rather than the cumulative performances over the study duration. Other differences between the current and previous studies include the fact that our previous analysis was performed on unnormalized data. In addition, a minimum of four rather than three scans were required for inclusion for the current study. Normalization was essential since CKDT staging is based on normalized GFR values. These differences between our previous and current studies could explain some of the disparities in conclusions. For example, previously we concluded that there was significant difference between measured and estimated unnormalized
GFR slopes. We now concluded in this current work that there was no significant difference between the normalized estimated and measured GFR slopes. Thus, our current results suggest a better performance of estimated slopes after normalization of the whole data set. Another related important finding is that, after normalization, the estimated variability in annual changes from both estimators for the whole data set was not significantly different from iGFR annual change. In our previous unnormalized GFR analyses we observed a significantly higher RMSE for iGFR slopes when compared to eGFR slopes. A lower RMSE for iGFR slopes in this analysis is a new finding of a larger cohort after normalization of the data set and thus adds another support for the use of normalized iGFR data in longitudinal analyses. In fact, after normalization the variability in iGFR results was lower than that from eGFR slopes. A new finding is the striking differences between $\Delta$ iGFR and $\Delta$ eGFR slopes in stage analyses (except MDRD-IDMS at stage 4). In addition, there was a universal overestimation of annual change when extrapolated from eGFR equations. We offer the conclusion that wide variations in \% annual changes across stages tend to off set each other and thus explain the superficial resemblances between measured and estimated slopes when all data set is lumped together. The variability (RMSE) in both measured and estimated slopes was also high and thus reflects the inherent limitation of the exponential analysis for the study of decline in renal transplant function over time when CKDT stages are considered. 
Determination of half-lives for each stage presented an opportunity to demonstrate the contrast between annual changes and half-life measurements. For example, the annual change estimated from CG equation for stage 1 was much lower than that in stage 4 while the half-life in stage 4 of 5.43 years was much lower than that of 36.6 years in stage 1 .

The quality of estimated slopes is related to the magnitude of the error in the annual change in transplant GFR. The larger the difference between the measured value and the true changes of GFR slopes, the poorer the quality of estimated slopes. Thus an important aim of this work was to determine the measurement of errors in the estimated slopes. We, therefore, quantified the measurement error between CKDT stages in the estimated slopes for each GFR estimator separately. A key finding was the striking differences between the estimated changes between the early stages (stage 1 and 2) and later stages (stage 3 and 4).

Measurement error in the whole data set for CG showed that $62 \%$ have errors less than or equal 1-fold. $9 \%$ had error less than or equal 2 -fold but more than 1-fold and $29 \%$ had more than 2 -fold. Analysis of errors in each stage subset showed different results. To complete our analysis the comparison between measurement errors for each estimator at the different CKDT stages was also performed. Only $12 \%$ of patients had error less than or equal 1 -fold in stage 1 while over $53 \%$ of patients in stage 4 demonstrated less than or equal to 1-fold difference from iGFR slope. Thus, the data demonstrates that measurement error in those with mild/moderate renal dysfunction is significantly larger than those with advanced form of CKDT. Thus, the results add another limitation to their clinical utility for the follow-up in patients with progressive CKDT. Collectively, these results support the detrimental impact of renal function on eGFR slope analysis. Our results are consistent with previous reports on the limited accuracy to predict changes in GFR. For example, of patients who lost GFR at rates faster than -1 $\mathrm{ml} / \mathrm{min} / 1.73 \mathrm{~m}^{2} /$ year, only $50 \%$ were correctly identified by the MDRD-IDMS slope as losing graft function. Moreover, White et al. [9] noted a varying accuracy to estimate GFR along K/DOQI stages. In addition, Tidman et al. [22] have reported limited accuracy of the current equations for identification of K/DOQI-suggested CKDT stages. Marriott et al. [23] compared the GFR estimated by the MDRD-IDMS and CG equations with 476 inulin clearances in 284 renal transplant recipients. They concluded that, in renal transplantation, the prediction performances of both CG and MDRD-IDMS equations ap- peared particularly impaired and potentially compromised the validity of the K/DOQI guidelines if implemented in their current form. Thus, estimation of rates of decline in GFR may be seriously hampered if point eGFR estimates are used to calculate eGFR slopes at different stages.

In spite of the detrimental effect of renal dysfunction in slope analysis, eGFR at first observation correlated significantly with that at last observation, confirming the notion $[24,25]$ that early kidney function is a useful surrogate marker for graft outcome.

\section{Limitations}

Firstly, we selected a patient population from one center. Independent observations by other centers would be helpful to support our conclusions. Secondly, GFR tests were not routinely performed on all patients on predetermined exact time points and therefore we cannot extrapolate actual or estimated rates of decline in kidney function in our centre. Because of lack of serial repeat iGFR in this study, we recognize that the progression of renal dysfunction in some of our patients could have occurred earlier than was identified at the time of their GFR. Patient attrition makes comparisons between intervals over time problematic [26]. Fourthly, we did not calibrate serum creatinine. A small systematic error in serum creatinine measurement can affect the results of a GFR [27]. Hallan's group showed previously that the bias due to a missing calibration decreases as serum creatinine increases. This is crucial since our cohort comprised a considerable percentage of patients with abnormal serum creatinine levels. Stevens et al. [28] suggested that creatinine calibration to MDRD-IDMS resolved most of the over- and underestimation of GFR when using the MDRD-IDMS formula without calibration. Despite these limitations this study has several advantages, including a large data set in which each patient has at least four consecutive iGFR tests.

\section{Conclusions}

In this precision performance study we estimated the variability of eGFR slopes and additionally the relative magnitudes of their imprecision across CKDT stages 1-4. CG and MDRD-IDMS estimated slopes significantly exaggerated graft loss in CKDT stages 1-4 except for stage 4 for MDRD-IDMS. The percentage of patients with 
measurement error of more than 1-fold in stage 1 for CG $(87.5 \%)$ and MDRD-IDMS (84.8\%) was significantly higher than that of CG (47.2\%) and MDRD-IDMS (47.2\%) for stage 4 , respectively. Thus the results suggest that transplant GFR slope prediction is affected by the degree of renal dysfunction. Errors in slope predictions are much higher in those with better renal function and thus add a major limitation for eGFR use in the longitudinal followup for patients with progressive CKDT.

\section{References}

1 Hsu CY, Chertow GM, Curhan GC: Methodological issues in studying the epidemiology of mild to moderate chronic renal insufficiency. Kidney Int 2002;61:1567-1576.

-2 Mariat C, Maillard N, Phayphet M, Thibaudin L, Laporte S, Alamartine E, Berthoux F: Estimated glomerular filtration rate as an end point in kidney transplant trial: where do we stand? Nephrol Dial Transplant 2008; 23:33-38.

3 Gassman JG, Greene T, Wright JT, et al: Design and statistical aspects of the African American Study of Kidney Disease and Hypertension (AASK). J Am Soc Nephrol 2003; 14:S154-S156.

4 Liang KY, Zeger SL: Longitudinal analysis using generalized linear models. Biometrics 1985;42:121-130.

5 Hariharan S, Kasiske B, Matas A, et al: Surrogate markers for long-term renal allograft survival. Am J Transplant 2004;4:1179.

-6 Levey A: Use of glomerular filtration measurements to assess the progression of renal disease. Semin Nephrol 1989;9:370-379.

7 Ravani P, Parfrey P, Murphy S, et al: Clinical research of kidney diseases IV: standard regression models. Nephrol Dial Transplant 2008;23:475-482.

-8 Aleksandra K, Madhurima A, Julio P, et al: CKD stage-to-stage progression in native and transplant kidney disease. Nephrol Dial Transplant 2008;23:693-700.

$\checkmark 9$ White C, Akbari A, Hussain N, et al: Chronic kidney disease stage in renal transplantation - classification using cystatin $\mathrm{C}$ and creatinine-based equations. Nephrol Dial Transplant 2007;22:3013-3020.

10 Gera M, Slezak JM, Rule AD, et al: Assessment of changes in kidney allograft function using creatinine-based estimates of glomerular filtration rate. Am J Transplant 2007;7: 880 .
11 Eriksen BO, Ingebretsen OC: In chronic kidney disease staging the use of the chronicity criterion affects prognosis and the rate of progression. Kidney Int 2007;72:1242-1248.

12 Gaspari F, Ferrari S, Stucchi N, et al: Performance of different prediction equations for estimating renal function in kidney transplantation. Am J Transplant 2004;4:18261835.

13 Bosma RJ, Doorenbos CR, Stegeman CA, et al: Predictive performance of renal function equations in renal transplant recipients: an analysis of patient factors in bias. Am J Transplant 2005;5:2193.

-14 Hossain MA, Zahran A, Emara M, Shoker A Accuracy to estimate rates of decline in glomerular filtration rate in renal transplant patients. Transplantation 2007;84:490-497.

15 Cockcroft DW, Gault MH: Prediction of creatinine clearance from serum creatinine. Nephron 1976;16:31-41.

16 Levey AS, Coresh J, Greene T, et al: Using standardized serum creatinine values in the modification of diet in renal disease study equation for estimating glomerular filtration rate. Ann Intern Med 2006;145:247254.

17 Perrone RD, Steinman TI, Beck GJ, et al: Utility of radioisotopic filtration markers in chronic renal insufficiency: simultaneous comparison of ${ }^{125}$ I-iothalamate, $169 \mathrm{yb}$ DTPA, 99mTc-DTPA and inulin. Am J Kidney Dis 1990;16:224-235.

18 Du Bois D, Du Bois EF: A formula to estimate the approximate surface area if height and weight be known. Arch Intern Med 1916;17: 863.

19 The Australian Creatinine Consensus Working Group: Chronic kidney disease and automatic reporting of estimated glomerular filtration rate: a position statement. Med J Aust 2005; 183:138-141.
20 Li Q, Racine JS: Nonparametric Econometrics: Theory and Practice. Princeton, Princeton University Press, 2007.

21 Parzen E: On estimation of a probability density function and mode. Ann Math Stat 1962;33:1065-1076.

22 Tidman M, Sjostrom P, Jones I: A comparison of GFR estimating formulae based upon s-cystatin C and s-creatinine and a combination of the two. Nephrol Dial Transplant 2008;23:154-160.

23 Mariat C, Alamartine E, Afiani A, et al: Predicting glomerular filtration rate in kidney transplantation: are the K/DOQI guidelines applicable? Am J Transplant 2005;5:26982703.

24 Hariharam S, McBride MA, Cherikh WS, et al: Post-transplant renal function in the first year predicts long-term kidney transplant survival. Kidney Int 2002;62:311.

25 Salvadori A, Rosati A, Bock A, et al: Estimated one-year glomerular filtration rate in the best predictor of long-term graft function following renal transplant. Transplantation 2006;81:202.

26 Kasiske BL, Gaston RS, Gourishankar S, et al: Long-term deterioration of kidney allograft function. Am J Transplant 2005;5: 1405-1414.

27 National Kidney Foundation: Kidney Disease Outcomes Quality Initiative Clinical Practice Guidelines for Chronic Kidney Disease: Evaluation, Classification, and Stratification. 5. Evaluation of Laboratory Measurements for Clinical Assessment of Kidney Disease. Guideline 4. Estimation of GFR. New York, National Kidney Foundation, 2000.

28 Stevens LA, Coresh J, Greene T, Levey AS: Assessing kidney function - measured and estimated glomerular filtration rate. $\mathrm{N}$ Engl J Med 2006;354:23. 